\title{
Asymptotics for functions associated with heat flow on the Sierpinski carpet
}

\author{
B.M. Hambly*
}

November 17, 2008

\begin{abstract}
We establish the asymptotic behaviour of the partition function, the heat content, the integrated eigenvalue counting function and, for certain points, the on-diagonal heat kernel of generalized Sierpinski carpets. For all these functions the leading term is of the form $x^{\gamma} \phi(\log x)$ for a suitable exponent $\gamma$ and $\phi$ a periodic function. We also discuss similar results for the heat content of affine nested fractals.
\end{abstract}

\section{Introduction}

The study of the relationship between the analytic and geometric properties of a bounded domain goes back at least to the celebrated Theorem of Weyl's showing that the volume of a domain could be recovered from the asymptotics of the eigenvalue counting function. Our interest here is in some related but slightly smoother functions, the integrated eigenvalue counting function, the partition function and the heat content. We will focus on determining the asymptotic behaviour of these functions on the Sierpinski carpet, an infinitely ramified fractal, for which few detailed properties are known.

For finitely ramified fractals there is a well developed theory concerning spectral asymptotics going back to [16] and in the physics literature to [29]. In the case of the Sierpinski gasket, using spectral decimation, it is possible to enumerate the eigenvalues of the Laplace operator and explicitly construct the eigenvalue counting function $N(\lambda)$. It can be shown [20] that, for either the Dirichlet or Neumann Laplacian, there is a $\log 5$-periodic function $\phi$ such that as $\lambda \rightarrow \infty$, the associated eigenvalue counting function $N(\lambda)=\lambda^{d_{s} / 2} \phi(\log \lambda)+O(1)$, where $d_{s}=$ $\log 3 / \log 5$ is the spectral dimension of the Sierpinski gasket. More generally, in the case of p.c.f. self-similar sets, there is a spectral dimension $d_{s}$ such that $0<\liminf _{\lambda \rightarrow \infty} N(\lambda) \lambda^{-d_{S} / 2} \leq$ $\lim \sup _{\lambda \rightarrow \infty} N(\lambda) \lambda^{-d_{S} / 2}<\infty$ and it is possible for the limit $N(\lambda) \lambda^{-d_{S} / 2}$ to exist. However, if the fractal has scale factors with logarithms that are rationally related, then there is a possibly periodic function which appears in the large $\lambda$ asymptotics, [21]. In the case of nested fractals this function was shown to be periodic due to the existence of strictly localized eigenfunctions in [8]. A further development due to [30] shows that the existence of localized eigenfunctions in this setting is related to the existence of certain critical points in the complex dynamics of an associated rational map.

As yet there are no corresponding results for infinitely ramified fractals such as the Sierpinski carpet. In this paper we will consider some closely related functions and show that there are possibly periodic functions which arise in their asymptotics. We do not have a proof that these

*Mathematical Institute, University of Oxford, 24-29 St Giles, Oxford OX1 3LB, UK.

E-mail: hambly@maths.ox.ac.uk 
periodic functions are non-constant. It should be noted that although the Laplace operator on generalized Sierpinski carpet was constructed in [2],[5] as the generator of Brownian motion on the carpet, there has only recently been progress on the question of the uniqueness of this Brownian motion [7]. It was previously shown that there is a self-similar process on the carpet and it this process with its associated Laplace operator that we use throughout the paper.

Let $F \subset \mathbb{R}^{D}$ be a bounded Sierpinski carpet or affine nested fractal. In order to define the heat content and partition function we will need to define the outer boundary of the set $F$. For each closed set $A$, let $\operatorname{Cov}(A)$ be the set of points covered by $A$, that is if we decompose $\mathbb{R}^{D} \backslash A$ into connected components $\left\{V_{j}\right\}_{j=1}^{\infty}$ and denote by $V_{U(A)}$ the unbounded component, then $\operatorname{Cov}(A)=\mathbb{R}^{2} \backslash V_{U(A)}$. We note that if the set $A$ has holes, these may be contained in $\operatorname{Cov}(A)$. The outer boundary of the fractal is defined to be $\partial F=\partial \operatorname{Cov}(F)$.

The partition function $Z_{F}(t)$ is defined to be

$$
Z_{F}(t)=\int_{F} p_{t}^{a}(x, x) \mu(d x),
$$

where $p_{t}^{a}(x, y)$ is the heat kernel for the Laplacian on the fractal with Dirichlet boundary conditions or equivalently the transition density for the corresponding diffusion process with absorption on $\partial F$. The measure $\mu$ is the $d_{f}$-dimensional Hausdorff measure on the fractal $F$ normalized so that $\mu(F)=1$.

We consider the short time asymptotics for the partition function, which is the Laplace transform of the eigenvalue counting measure and show that there is a periodic function which appears in the asymptotics of $Z_{F}$. If this periodic function is indeed constant we could use a Tauberian theorem to recover the existence of the limit for the normalized eigenvalue counting function $N_{F}$ (for the Dirichlet Laplacian). However, with the result we obtain here, we cannot obtain a deeper result for $N_{F}(\lambda)$ on the carpet than the existence of constants $c_{1}, c_{2}$ such that

$$
c_{1} \lambda^{d_{s} / 2} \leq N_{F}(\lambda) \leq c_{2} \lambda^{d_{s} / 2},
$$

a result easily deduced from the transition density estimates of [5]. We will consider the eigenvalue problem directly but will have to consider a smoother function than $N_{F}$ itself. We will be able to produce an oscillatory function in the high frequency asymptotics for the integrated eigenvalue counting function.

The heat content of the fractal $F$ is the total heat energy in the set at a given time due to unit boundary conditions and zero initial conditions within the set. We begin with a more general problem. Let $\phi: \partial F \rightarrow[0, \infty)$ be bounded and measurable. We write $\Delta_{F}$ for the Laplace operator on $F$ and consider the following partial differential equation in $F$. Let $u: F \times[0, \infty) \rightarrow[0, \infty)$ satisfy

$$
\begin{aligned}
\frac{\partial u}{\partial t} & =\Delta_{F} u, \quad x \in F \backslash \partial F, t>0, \\
u(x, 0) & =0, \quad x \in F \backslash \partial F, \\
u(x, t) & =\phi(x), \quad x \in \partial F, t>0 .
\end{aligned}
$$

This equation has a probabilistic representation for its solution. Let $\left\{X_{t}\right\}$ be the stochastic process with generator $\Delta_{F}$ on the set $F$ and let $T_{A}=\inf \left\{t \geq 0: X_{t} \in A\right\}$. Then the solution to (1.1) can be written as

$$
u(x, t)=\mathbb{E}_{x}\left(\phi\left(X_{T_{\partial F}}\right) I_{\left\{T_{\partial F}<t\right\}}\right) .
$$


The heat content $E_{F}(t)$ of $F$ at time $t$ is given by setting $\phi(x)=1$ for all $x \in \partial F$ and defining

$$
E_{F}(t)=\int_{F} u(x, t) \mu(d x)
$$

Our aim is to investigate for compact fractals the short time asymptotics of $E_{F}(t)$ when $\phi(x)=1$ for all $x \in \partial F$ or sometimes $\phi(x)=1$ for all $x \in A \subset \partial F$ and $\phi(x)=0$ for $x \in F \backslash A$ for some subset $A$ of the outer boundary. Our techniques are strongly influenced by [12] where comparison estimates for fractal boundary problems are established. More detailed results for the heat content of domains with fractal boundary can be found in $[15,10,11,13]$. In our setting we have a fractal domain with a boundary that may be piecewise smooth or fractal.

We also discuss the on-diagonal transition density (heat kernel) and consider its behaviour at certain periodic points within the fractal. There have been many papers which estimate this quantity uniformly over the set but here we are able to obtain sharper results at these particular points using the self-similar structure of the set.

We state our results for the two-dimensional Sierpinski carpet, a set of Hausdorff dimension $d_{f}=\log 8 / \log 3$ (shown in figure 1 ), with boundary given by the unit square. Let $\tau$ denote the time scale factor for the Sierpinski carpet, a constant for which there is no explicit formula (for estimates, see [3]), and define the walk dimension $d_{w}=\log \tau / \log 3$.

Theorem 1.1 There exists a $\log \tau$-periodic function $\psi_{1}(t)$ such that as $t \downarrow 0$,

$$
Z_{F}(t)=t^{-d_{f} / d_{w}} \psi_{1}(-\log t)+O\left(t^{-1 / d_{w}}\right) .
$$

There exists a $\log \tau$-periodic function $\psi_{2}(\lambda)$ such that as $\lambda \rightarrow \infty$,

$$
\int_{0}^{\lambda} N_{F}(s) d s=\lambda^{1+d_{f} / d_{w}} \psi_{2}(\log \lambda)+O\left(\lambda^{1+1 / d_{w}}\right) .
$$

There exists a $\log \tau$-periodic function $\psi_{3}(t)$ such that as $t \downarrow 0$,

$$
E_{F}(t)=t^{\left(d_{f}-1\right) / d_{w}} \psi_{3}(-\log t)+O\left(t^{d_{f} / d_{w}}\right)
$$

There exists a $\log \tau$-periodic function $\psi_{4}(t)$ such that, for points $x \notin \partial F$ which are fixed points of some finite combination of the contraction maps that define the carpet, as $t \downarrow 0$,

$$
p_{t}^{a}(x, x)=t^{-d_{f} / d_{w}} \psi_{4}(-\log t)+O\left(\exp \left(-c t^{-1}\right)\right) .
$$

The generic result is that a symmetric self-similar set with the same scale factors will have $Z_{F}(t)=t^{-d_{f} / d_{w}}(\psi(-\log t)+o(1))$ and $E_{F}(t)=t^{\left(d_{f}-d_{b}\right) / d_{w}}(\phi(-\log t)+o(1))$, where $d_{b}$ is the dimension of the boundary and $\psi, \phi$ are suitable periodic functions. A variety of such results are given in Theorems 4.1, 4.2, 5.2, 5.4 and 5.6. For the case of the partition function recent work in [19] gives a generalization to the case where the measure is not self-similar. In the affine nested fractal case we show an example where the periodic function will be a constant if the set does not have commensurate resistance scaling ratios.

The outline of the paper is as follows. In Section 2 we will establish two comparison lemmas which are the main technical tools required to prove our results. They will be derived for fractional diffusions on fractional metric spaces which will allow us to apply them to Sierpinski carpets as well as to affine nested fractals. In Sections 3 and 4 we consider generalized Sierpinski carpets 
and, by exploiting the existence of a self-similar diffusion on the carpet, show how the short time asymptotics of both the partition function and the heat content can be reduced to a renewal equation. Finally, in Section 5, we will give some examples to show the range of possible behaviour of these functions for affine nested fractals. We note that throughout the paper we will write $c$ for a generic constant whose value may be different at each appearance, while $c_{i}$ will have a fixed value within a given proof but will vary between proofs.

Acknowledgement: The author would like to thank Jun Kigami and Christophe Sabot for helpful comments and Naotaka Kajino for a careful reading of an earlier version.

\section{The comparison lemmas}

The key results needed for the discussion are comparison lemmas. For the heat content such a comparison is initially due to [12] in the case of a bounded domain in $\mathbb{R}^{D}$. We will establish these results for a fractional diffusion in a bounded fractal domain.

We begin by recalling the definition of a fractional diffusion from [1]. Firstly we define a fractional metric space.

Definition 2.1 Let $(F, d)$ be a complete metric space and $\mu$ a Borel measure on $(F, \mathcal{B}(F))$. We call $(F, d, \mu)$ a fractional metric space if

1. $(F, d)$ has the midpoint property.

2. There exists $d_{f}>0$ and constants $c_{1}, c_{2}$ such that if $r_{0} \in(0, \infty]$ is the diameter of $F$, then

$$
c_{1} r^{d_{f}} \leq \mu(B(x, r)) \leq c_{2} r^{d_{f}}, \quad x \in F, 0<r \leq r_{0},
$$

where $B(x, r)=\{y: d(x, y) \leq r\}$.

Definition 2.2 A fractional diffusion $F D\left(d_{f}, d_{w}\right)$ on a fractional metric space $(F, d, \mu)$ is a Markov process $X=\left(P^{x}, x \in F, X_{t}, t \geq 0\right)$ with the properties

1. $X$ is a conservative diffusion with state space $F$.

2. $X$ is $\mu$-symmetric.

3. $X$ has a symmetric transition density $p_{t}(x, y)=p_{t}(y, x), t>0, x, y \in F$ which satisfies the Chapman-Kolmogorov equations and is, for each $t>0$, jointly continuous.

4. There exist constants $c_{1}, c_{2}, c_{3}, c_{4}, t_{0}=r_{0}^{d_{w}}$ such that $\forall x, y \in F, 0<t \leq t_{0}$,

$$
\begin{gathered}
c_{1} t^{-d_{f} / d_{w}} \exp \left(-c_{2}\left(\frac{d(x, y)^{d_{w}}}{t}\right)^{1 /\left(d_{w}-1\right)}\right) \leq p_{t}(x, y) \\
\leq c_{3} t^{-d_{f} / d_{w}} \exp \left(-c_{4}\left(\frac{d(x, y)^{d_{w}}}{t}\right)^{1 /\left(d_{w}-1\right)}\right)
\end{gathered}
$$

The exit time from a set $A$ is the hitting time for the complement $A^{c} ; T_{A^{c}}=\inf \left\{t \geq 0: X_{t} \in\right.$ $\left.A^{c}\right\}$. The distance from a point to a set is defined to be $d(x, A)=\inf _{y \in A} d(x, y)$. 
Lemma 2.3 Let $A \subset F$ be a closed set. There exist constants $c_{1}, c_{2}$ such that

$$
P_{x}\left(T_{A^{c}}<t\right) \leq c_{1} \exp \left(-c_{2}\left(\frac{d\left(x, A^{c}\right)^{d_{w}}}{t}\right)^{1 /\left(d_{w}-1\right)}\right) .
$$

Proof: In [1] Equation (3.21) it is shown that for fractional diffusions there are constants $c, \tilde{c}$ such that

$$
P^{x}\left(T_{B(x, r)^{c}}<t\right) \leq c \exp \left(-\tilde{c}\left(\frac{r^{d_{w}}}{t}\right)^{1 /\left(d_{w}-1\right)}\right), \forall x \in F, 0<t \leq t_{0}, 0<r \leq r_{0} .
$$

If $r^{\prime}=\sup \{r: B(x, r) \subset A\}$, then $r^{\prime}=d\left(x, A^{c}\right)$. Thus, as

$$
P^{x}\left(T_{A^{c}}<t\right) \leq P^{x}\left(T_{B\left(x, r^{\prime}\right)^{c}}<t\right),
$$

applying (2.1) gives the result.

We consider the fractional metric space $F$ to be bounded and we denote its boundary by $\partial F$. We write $p_{t}^{a}(x, y)$ for the transition density with respect to $\mu$ of the diffusion on $F$ with absorption on $\partial F$ and $p_{t}(x, y)$ for the transition density with respect to $\mu$ of the diffusion with reflection on $\partial F$. It is elementary that $p_{t}^{a}(x, y) \leq p_{t}(x, y)$ for all $x, y \in F, t>0$. Let $p_{t}^{a, A}(x, y)$ be the transition density for the diffusion which is absorbed on $\partial F$ and on the closed set $A$.

For the sets $F$ we consider here the transition densities $p_{t}^{a}(x, y), p_{t}^{a, A}(x, y)$ for $X$ absorbed at the boundary and on $A$ will be jointly continuous in $x, y$ for $t>0$. We will assume this for obtaining our comparison results for fractional diffusions.

\subsection{The transition density}

Let $C$ be a closed subset of $F$. The main comparison lemma is the following:

Lemma 2.4 There exist positive constants $c_{1}, c_{2}, c_{3}, t_{0}$ with $c_{3}<1$ such that for all $x \in F$ and $0<t<t_{0}$ we have

$$
p_{t}^{a, C}(x, x) \leq p_{t}^{a}(x, x) \leq p_{t}^{a, C}(x, x)+c_{1} t^{-d_{f} / d_{w}} \min \left(\exp \left(-c_{2}\left(\frac{d(x, C)^{d_{w}}}{t}\right)^{1 /\left(d_{w}-1\right)}\right), c_{3}\right) .
$$

Proof: We begin by making the observation that if $X^{a}$ denotes the fractional diffusion on $F$ with absorption on $\partial F$, then

$$
\begin{aligned}
p_{t}^{a, C}(x, x) & =\lim _{r \downarrow 0} \frac{P^{x}\left(X_{t}^{a} \in B_{r}(x)\right)}{\mu\left(B_{r}(x)\right)}, \\
p_{t}^{a, C}(x, x) & =\lim _{r \downarrow 0} \frac{P^{x}\left(X_{t}^{a} \in B_{r}(x), T_{C}>t\right)}{\mu\left(B_{r}(x)\right)},
\end{aligned}
$$

and

$$
P^{x}\left(X_{t}^{a} \in B_{r}(x)\right)=P^{x}\left(X_{t}^{a} \in B_{r}(x), T_{C}>t\right)+P^{x}\left(X_{t}^{a} \in B_{r}(x), T_{C} \leq t\right) .
$$

The left hand inequality of (2.2) follows from the first term of (2.3). 
We show that there is a constant $c_{4}$ such that if $d(x, C)>c_{4} t^{1 / d_{w}}$, then

$$
p_{t}^{a}(x, x) \leq p_{t}^{a, C}(x, x)+c_{1} t^{-d_{f} / d_{w}} \exp \left(-c_{2}\left(\frac{d(x, C)^{d_{w}}}{t}\right)^{1 /\left(d_{w}-1\right)}\right) .
$$

To establish (2.4) we take $x \in F$ and choose $r<d(x, C) / 2$. We need to estimate

$$
\begin{aligned}
P^{x}\left(X_{t}^{a} \in B_{r}(x), T_{C} \leq t\right) & =\int_{0}^{t} \int_{C} P^{x}\left(X_{t}^{a} \in B_{r}(x) \mid T_{C} \in d s, X_{T_{C}} \in d z\right) P^{x}\left(T_{C} \in d s, X_{T_{C}} \in d z\right) \\
& =\int_{0}^{t} \int_{C} P^{z}\left(X_{t-s}^{a} \in B_{r}(x)\right) P^{x}\left(T_{C} \in d s, X_{T_{C}} \in d z\right) \\
& \leq \sup _{z \in C, y \in B_{r}(x), 0 \leq s \leq t} p_{s}^{a}(z, y) \mu\left(B_{r}(x)\right) P^{x}\left(T_{C} \leq t\right) \\
& \leq \sup _{z \in C, y \in B_{r}(x), 0 \leq s \leq t} p_{s}^{a}(z, y) \mu\left(B_{r}(x)\right) .
\end{aligned}
$$

We recall that by definition of a fractional diffusion there exist constants $c_{5}, c_{6}, t_{0}$ such that for all $x, y \in F$ and $s<t_{0}$,

$$
p_{s}(z, y) \leq c_{5} s^{-d_{f} / d_{w}} \exp \left(-c_{6}\left(\frac{d(z, y)^{d_{w}}}{s}\right)^{1 /\left(d_{w}-1\right)}\right) .
$$

Let $c_{4}=2\left(d_{f}\left(d_{w}-1\right) / d_{w} c_{6}\right)^{1-1 / d_{w}}$. Now, if $d(x, C)>c_{4} t^{1 / d_{w}}$, then

$$
\xi=d\left(B_{r}(x), C\right)=\inf _{y \in B_{r}(x), z \in C} d(y, z)>\frac{1}{2} d(x, C)>\frac{1}{2} c_{4} t^{1 / d_{w}} .
$$

By (2.5), for $z \in C, y \in B_{r}(x)$ we have

$$
p_{s}(z, y) \leq f(s):=c_{5} s^{-d_{f} / d_{w}} \exp \left(-c_{6}\left(\frac{\xi^{d_{w}}}{s}\right)^{1 /\left(d_{w}-1\right)}\right) .
$$

Our choice of $c_{4}$, such that $\xi>\frac{1}{2} c_{4} s^{1 / d_{w}}$ for $0<s<t$, ensures that

$$
f^{\prime}(s)=s^{-1} f(s)\left(-\frac{d_{f}}{d_{w}}+\frac{c_{6}}{d_{w}-1} s^{-1 /\left(d_{w}-1\right)} \xi^{d_{w} /\left(d_{w}-1\right)}\right),
$$

is positive and hence the bound $f(s)$ is a non-decreasing function over $0 \leq s \leq t$. Thus, as $p_{s}^{a}(z, y) \leq p_{s}(z, y)$ for all $s, z, y$, we have

$$
\sup _{z \in C, y \in B_{r}(x), 0 \leq s \leq t} p_{s}^{a}(z, y) \leq c_{5} t^{-d_{f} / d_{w}} \exp \left(-c_{6}\left(\frac{\xi^{d_{w}}}{t}\right)^{1 /\left(d_{w}-1\right)}\right) .
$$

Letting $r \downarrow 0$ so that $\xi \uparrow d(x, C)$ we have the required upper bound for (2.4).

As we have a fractional diffusion, we know that $p_{t}^{a}(x, x) \leq p_{t}(x, x) \leq c_{5} t^{-d_{f} / d_{w}}$ for $t<t_{0}$. Hence, as $p_{t}^{a, C}(x, x)>0$, it is straightforward to see that,

$$
p_{t}^{a}(x, x) \leq p_{t}^{a, C}(x, x)+c_{5} t^{-d_{f} / d_{w}} .
$$

Combining this with (2.4) we set $c_{1}=c_{5} e^{c_{6} c_{4}^{d_{w} /\left(d_{w}-1\right)}}, c_{2}=c_{6}, c_{3}=e^{-c_{6} c_{4}^{d_{w} /\left(d_{w}-1\right)}}$ to get the result. 
Remark 2.5 We note that the above proof can be adapted to compare the transition density for the reflected and the absorbed processes. As already noted $p_{t}^{a}(x, y) \leq p_{t}(x, y)$. We replace $p^{a}$ by $p$ and $p^{a, C}$ by $p^{a}$ and will obtain the existence of suitable constants such that for all $0<t<1$ and $x \in F$,

$$
p_{t}(x, x) \leq p_{t}^{a}(x, x)+c_{1} t^{-d_{f} / d_{w}} \min \left\{\exp \left(-c_{2}\left(\frac{d(x, \partial F)^{d_{w}}}{t}\right)^{1 /\left(d_{w}-1\right)}\right), c_{3}\right\}
$$

\subsection{The heat content}

Let $C \subset F$ be a closed subset. We fix the temperature of $C$ to be 0 while $\partial F \backslash C$ is maintained at temperature $\phi$.

Let $\tilde{F}=F \backslash C$ and $\tilde{u}: \tilde{F} \times[0, \infty) \rightarrow[0, \infty)$ be the weak solution to

$$
\begin{aligned}
\frac{\partial \tilde{u}}{\partial t} & =\Delta_{F} \tilde{u}, \quad x \in \tilde{F} \\
\tilde{u}(x, 0) & =0, \quad x \in \tilde{F} \backslash \partial \tilde{F}, \\
\tilde{u}(x, t) & =0, \quad x \in C, t>0, \\
\tilde{u}(x, t) & =\phi(x), \quad x \in \partial F \backslash C, t>0 .
\end{aligned}
$$

Let

$$
S=c l\{x \in \partial F: \phi(x) \neq 0\}
$$

Lemma 2.6 There exist constants $c_{1}, c_{2}, c_{3}$ such that

$$
0 \leq u(x, t)-\tilde{u}(x, t) \leq c_{1}\|\phi\|_{\infty} \exp \left(-c_{2}\left(\frac{d(x, S)^{d_{w}}}{t}\right)^{1 /\left(d_{w}-1\right)}-c_{3}\left(\frac{d(x, C)^{d_{w}}}{t}\right)^{1 /\left(d_{w}-1\right)}\right)
$$

for all $x \in F, t>0$.

Proof: This follows exactly the same argument as the proof of Theorem 1.1 in [12], where we replace their Brownian motion $B$ by our fractional diffusion $X$, to derive their equation $(2.7)$

$$
\tilde{u}(x, t) \leq u(x, t) \leq \tilde{u}(x, t)+\|\phi\|_{\infty} \mathbb{P}_{x}\left(T_{S}<t\right)^{1 / 2} \mathbb{P}_{x}\left(T_{C}<t\right)^{1 / 2} .
$$

We can now apply Lemma 2.3 to obtain the result.

Let $\tilde{E}_{F}=\int_{\tilde{F}} \tilde{u}(x, t) \mu(d x)$ be the associated heat content.

Corollary 2.7 We assume that $\mu(C)=0$ and that there is a c such that

$$
\int_{F} \exp \left(-c\left(d(x, S)^{d_{w}} / t\right)^{1 /\left(d_{w}-1\right)}\right) \mu(d x)<\infty .
$$

Then

$$
E_{F}(t)-\tilde{E}_{F}(t) \leq c_{1}\|\phi\|_{\infty} \int_{F} \exp \left(-c_{2}\left(\frac{d(x, S)^{d_{w}}}{t}\right)^{1 /\left(d_{w}-1\right)}-c_{3}\left(\frac{d(x, C)^{d_{w}}}{t}\right)^{1 /\left(d_{w}-1\right)}\right) \mu(d x),
$$

for all $t>0$.

Proof: We integrate the estimate (2.8) and use the fact that $C$ has $\mu$-measure 0 . 


\section{The Sierpinski carpet}

We will work with $F$ a generalized Sierpinski carpet as defined in [5]. Let $D \geq 2$ and set $F_{0}=[0,1]^{D}$. Let $l_{F} \in \mathbb{N}$ with $l_{F} \geq 3$ be the length scale factor. Let $\mathcal{S}_{n}$ denote the set of cubes of side $l_{F}^{-n}$ contained in $F_{0}$. Now take $m_{F} \in \mathbb{N}$ with $1 \leq m_{F} \leq l_{F}^{D}$ and let $F_{1}$ be the union of $m_{F}$ distinct elements of $\mathcal{S}_{1}$. We make the following assumptions on $F_{1}$. Note that the third assumption differs from that used in [2,5], a point which is discussed in [7].

Assumption 3.1 1. $F_{1}$ is preserved by all of the isometries of the unit cube $F_{0}$.

2. Int $\left(F_{1}\right)$ is connected and contains a path connecting the hyperplanes $\left\{x_{1}=0\right\}$ and $\left\{x_{1}=1\right\}$.

3. Let $n \geq 1$ and $B$ be a cube in $F_{0}$ of side length $2 l_{F}^{-n}$ which is the union of $2^{D}$ distinct elements of $\mathcal{S}_{n}$. Then, if $\operatorname{Int}\left(F_{1}\right) \cap B \neq \emptyset$, then it is connected.

4. $F_{1}$ contains the line segment $\left\{0 \leq x_{1} \leq 1, x_{2}=0, \ldots, x_{d}=0\right\}$.

There exist maps $\left\{\psi_{1}, \ldots, \psi_{m_{F}}\right\}$, where the $\psi_{i}$ are similitudes of contraction factor $l_{F}$, taking the unit cube to the $m_{F}$ subcubes of $F_{1}$. We now consider the decreasing sequence of sets $F_{n}=\cup_{i=1}^{m_{F}} \psi_{i}\left(F_{n-1}\right)$ and obtain the set $F$ as

$$
F=\bigcap_{n=0}^{\infty} F_{n}
$$

This is a fractal set with Hausdorff dimension $\log m_{F} / \log l_{F}$.

We could consider the natural geodesic metric $d$ on the carpet, but we will just work in the Euclidean metric as these are equivalent under our Assumption 3.1(4) by [5]. We write $\mu$ for the $d_{f}$-dimensional Hausdorff measure on $F$. We note that $(F,||,. \mu)$ is a fractional metric space. Note that the boundary $\partial F$ of the fractal as a subset of $\mathbb{R}^{D}$ could be a fractal itself.
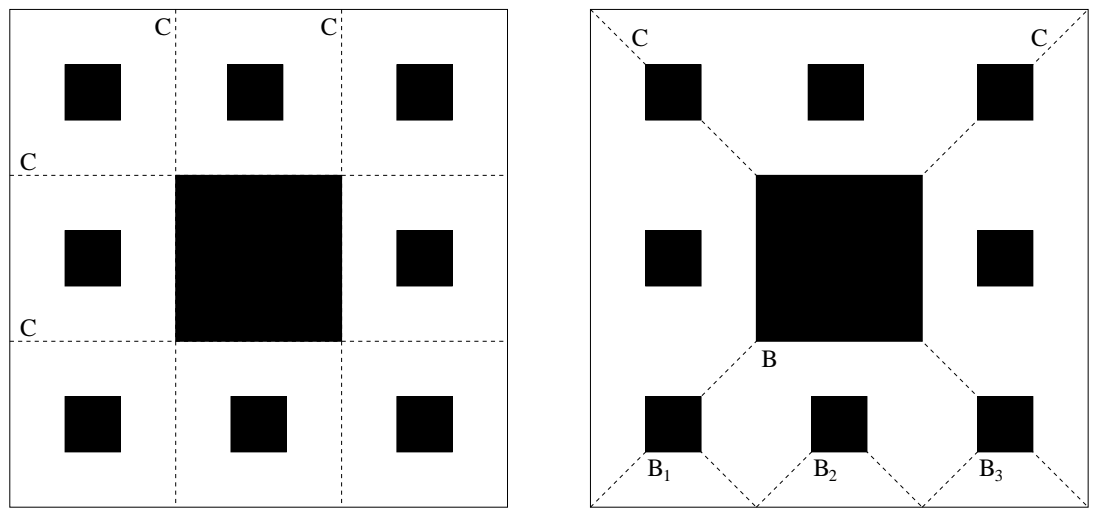

Figure 1: The Sierpinski carpet and its decompositions

The existence and properties of a canonical diffusion on the Sierpinski carpet has been the subject of much research [2], [4], [24], [5]. A major open question has been the uniqueness of this Brownian motion and progress has recently been made in [7]. We begin with a Theorem stating the results we will need to prove our main result. We first note that there is a constant $\rho_{F}$ which is determined by the scaling in the resistance of the Sierpinski carpet. We then define $\tau_{F}=m_{F} \rho_{F}$ to be the time scaling factor and then the walk dimension is $d_{w}=\log \tau_{F} / \log l_{F}$. For a set $A \subset F$ we write $\psi_{i}(A)=\left\{\psi_{i}(x): x \in A\right\}$ for the set scaled by the similitude $\psi_{i}$. 
Theorem 3.2 (a) There exists a local regular Dirichlet form $(\mathcal{E}, \mathcal{F})$ on $L^{2}(F, \mu)$ satisfying the self-similarity property

$$
\mathcal{E}(f, g)=\sum_{i=1}^{m_{F}} \rho_{F} \mathcal{E}\left(f \circ \psi_{i}, g \circ \psi_{i}\right) .
$$

(b) The corresponding stochastic process $\left\{X_{t}: t \geq 0\right\}$ on $F$ satisfies the scaling law that for any similitude $\psi_{i}, A \subset F, x \in A$ the law of $\left\{\psi_{i}\left(X_{t}\right): 0 \leq t \leq T_{A}\right\}$ under $\mathbb{P}^{x}$ is equal to the law of $\left\{X_{t / \tau_{F}}: 0 \leq t \leq T_{\psi_{i}(A)}\right\}$ under $\mathbb{P}^{\psi_{i}(x)}$.

(c) There exists a jointly continuous transition density $p_{t}(x, y)$ which satisfies the sub-Gaussian bounds, for all $x, y \in F$ and $t \geq 0$,

$$
c_{1} t^{-d_{f} / d_{w}} \exp \left(-c_{2}\left(\frac{|x-y|^{d_{w}}}{t}\right)^{1 /\left(d_{w}-1\right)}\right) \leq p_{t}(x, y) \leq c_{3} t^{-d_{f} / d_{w}} \exp \left(-c_{4}\left(\frac{|x-y|^{d_{w}}}{t}\right)^{1 /\left(d_{w}-1\right)}\right) .
$$

(d) The diffusion $X$ with absorption on $\partial F \cup C$ for a closed set $C$ has a transition density $p_{t}^{a, C}(x, y)$ which is jointly continuous.

Proof: (a) The original construction of a Brownian motion on $F$ for $D=2$ was given in [2], though the resulting diffusion was not shown to be self-similar. The existence of a self-similar diffusion was established in [24]. In [4] the construction was extended to $D \geq 3$ and a self-similar process can be constructed following the technique of [24] as remarked in [4] Remark 5.11.

(b) is a straightforward consequence of (a).

(c) The original transition density estimates on the carpet were obtained in [4]. Now that the Brownian motion on the Sierpinski carpet is known to be unique, these estimates will hold for the unique self-similar diffusion on the carpet.

(d) This result, when $C=\emptyset$, is given in Proposition 6.15 in [5]. The same arguments can be applied to deduce the result in the more general setting.

Corollary 3.3 The self-similar diffusion $X$ on the Sierpinski carpet is a $F D\left(d_{f}, d_{w}\right)$.

From this point on we will just work with the self-similar diffusion on the carpet and refer to it as the Brownian motion on the carpet. We also need to show that we have joint continuity for the transition density with absorption.

In order to define the heat content that we consider here we need to define an appropriate $\phi$ on $\partial F$. We do this by setting $\phi(x)=1$ for $x \in \partial F \cap \partial[0,1]^{D}$ and $\phi(x)=0$ for $x \in \partial F \backslash \partial[0,1]^{D}$. Thus if a cube has been removed from the 'interior' of a face we do not consider the full boundary of the set in $\mathbb{R}^{D}$ but just the part of the boundary that intersects the boundary of the unit cube in $\mathbb{R}^{D}$. Think of this as pressing the face of $F$ against a hyperplane held at temperature 1 . The heat content $E_{F}(t)$ of the carpet is then defined to be the corresponding integrated solution to the original PDE, (1.1).

Let the number of hypercubes of $F_{1}$ which intersect with a $D$-1-dimensional face of $\partial[0,1]^{D}$ be $b_{1}$. We write $d_{b_{1}}=\log b_{1} / \log l_{F}$ for the dimension of the intersection of the boundary of the fractal with a $D-1$ dimensional face of the unit cube. We also define $b_{2}$ to be the number of hypercubes of $F_{1}$ which intersect with a $D-2$ dimensional face of $\partial[0,1]^{D}$ and let $d_{b_{2}}=\log b_{2} / \log l_{F}$ denote the dimension of this boundary. By construction we have $d_{f}>d_{b_{1}}>d_{b_{2}}$. If $D=2$ then, by Assumption 3.14 , we have $d_{b_{1}}=1$, and we must also have $d_{b_{2}}=0$. For $D=3$ we must, by 
Assumption 3.14 , have $d_{b_{2}}=1$. Note that Assumption 3.14 is not essential for the existence of a process and can be dropped [17].

The fundamental result we need is a scaling for both the partition function and the heat content.

Lemma 3.4 The partition function for the Brownian motion on the Sierpinski carpet satisfies

$$
Z_{\psi_{i}(F)}(t)=Z_{F}\left(\tau_{F} t\right), \quad \forall i=1, \ldots, m_{F} .
$$

The heat content for the Brownian motion on the Sierpinski carpet satisfies

$$
E_{\psi_{i}(A)}(t)=\frac{1}{m_{F}} E_{A}\left(\tau_{F} t\right), \quad \forall i=1, \ldots, m_{F} .
$$

Proof: This is a simple application of the scaling of the diffusion and the definitions. For the partition function we use the scaling in the transition density arising from Theorem 3.2 (b) to see that

$$
P^{x}\left(X_{t} \in B_{r}(x), t<T_{\partial F}\right)=P^{\psi_{i}(x)}\left(X_{t / \tau_{F}} \in \psi_{i}\left(B_{r}(x)\right), t / \tau_{F}<T_{\psi_{i}(\partial F)}\right) .
$$

By dividing by $\mu\left(B_{r}(x)\right)$ and taking the limit as $r \downarrow 0$ we arrive at

$$
p_{t}^{a}(x, x)=\frac{1}{m_{F}} p_{t / \tau_{F}}^{a, \psi_{i}(\partial F)}\left(\psi_{i}(x), \psi_{i}(x)\right), \quad \forall x \in F \backslash \partial F, t>0 .
$$

Thus we have

$$
\begin{aligned}
Z_{\psi_{i}(F)}(t) & =\int_{\psi_{i}(F)} p_{t}^{a, \psi_{i}(\partial F)}(x, x) \mu(d x) \\
& =\int_{\psi_{i}(F)} p_{\tau_{F} t}^{a}\left(\psi_{i}^{-1}(x), \psi_{i}^{-1}(x)\right) \mu\left(d \psi_{i}^{-1}(x)\right) \\
& =\int_{F} p_{\tau_{F} t}^{a}(x, x) \mu(d x)=Z_{F}\left(\tau_{F} t\right)
\end{aligned}
$$

For the heat content, note that by the scaling given in Theorem 3.2 (b) we have

$$
P^{x}\left(T_{A}<t\right)=P^{\psi_{i}(x)}\left(T_{\psi_{i}(A)}<t / \tau_{F}\right)
$$

Now integrate this and change variable in the same way as for the partition function to get the result.

We now state what could be considered as a simple version of the renewal theorem as given in [26], with error estimate as in [20] Theorem B.4.3.

Lemma 3.5 Let $f$ be a measurable function on $\mathbb{R}$ with $f(u) \rightarrow 0$ as $u \rightarrow-\infty$ which satisfies

$$
f(u)=f(u-\sigma)+g(u)
$$

for some piecewise continuous function $g$. If $|g(u)| \leq c_{1} e^{-c_{2}|u|}$ for some constants $c_{1}, c_{2}>0$ for all $u \in \mathbb{R}$, then there is a $\sigma$-periodic function $\theta$ such that

$$
\lim _{n \rightarrow \infty} f(u+n \sigma)=\theta(u)=\sum_{j=-\infty}^{\infty} g(u-j \sigma) .
$$

Also, as $u \rightarrow \infty$, we have

$$
|f(u)-\theta(u)|=O\left(e^{-c_{2} u}\right)
$$


Remark 3.6 It is easy to see that if $|g(u)| \leq c_{1} \exp \left(-c_{2} e^{c_{3}|u|}\right)$, then we have the same conclusion with

$$
|f(u)-\theta(u)|=O\left(e^{-c_{2} e^{c_{3} u}}\right)
$$

as $u \rightarrow \infty$.

We also need a lemma on the long time behaviour of the transition density for the absorbing process.

Lemma 3.7 There exists a $c_{0}, \lambda_{0}>0$ such that

$$
\begin{array}{r}
\sup _{x, y} p_{t}^{a}(x, y) \leq c_{0} \exp \left(-\lambda_{0} t\right), \quad t>1, \\
E_{F}(t) \geq 1-c_{0} e^{-\lambda_{0} t}, \quad t>1 .
\end{array}
$$

Proof: We note that from [5] Proposition 6.15 there is a discrete spectrum and a uniformly convergent eigenvalue expansion for the transition density with absorption. It is not difficult to show that the bottom eigenvalue of the Dirichlet spectrum is strictly positive and hence we have (3.3).

To establish (3.4) we observe that

$$
\begin{aligned}
1-E_{F}(t) & =\int_{F} P^{x}\left(T_{\partial F}>t\right) \mu(d x) \\
& =\int_{F} \int_{F} p_{t}^{a}(x, y) \mu(d y) \mu(d x) .
\end{aligned}
$$

Now use (3.3) to see that for $t>1$ we have $1-E_{F}(t) \leq c_{0} \exp \left(-\lambda_{0} t\right)$.

\section{The asymptotics}

\subsection{The heat content and partition function}

We can now state and prove our main results.

Theorem 4.1 Let $F$ be a generalized Sierpinski carpet in $\mathbb{R}^{D}$. There exists a periodic function $\phi_{p}$ such that as $t \rightarrow 0$, the partition function satisfies

$$
Z_{F}(t)=t^{-d_{s} / 2} \phi_{p}(-\log t)+O\left(t^{-d_{b_{1}} / d_{w}}\right) .
$$

Proof: For the partition function we define the set $C$ to be the at most $\left(l_{F}-1\right)^{D}$ hyperplanes which divide the carpet up into the $m_{F}$ subcubes of $F_{1}$. The two dimensional case is shown on the left of Figure 1 and consists of the four line segments from $(1 / 3,0)$ to $(1 / 3,1),(2 / 3,0)$ to $(2 / 3,1),(0,1 / 3)$ to $(1,1 / 3)$, and $(0,2 / 3)$ to $(1,2 / 3)$, which disconnect the level one carpet into its eight scaled copies. Let $\tilde{Z}_{F}(t)=\int_{F} p_{t}^{a, C}(x, x) \mu(d x)$. Then, by $(3.1)$,

$$
\begin{aligned}
\tilde{Z}_{F}(t) & =\sum_{i=1}^{m_{F}} \int_{\psi_{i}(F)} p_{t}^{a, C}(x, x) \mu(d x) \\
& =\sum_{i=1}^{m_{F}} Z_{\psi_{i}(F)}(t) \\
& =m_{F} Z_{F}\left(\tau_{F} t\right) .
\end{aligned}
$$


We now apply our comparison Lemma 2.4. Firstly, by (2.2) it is clear that $\tilde{Z}_{F}(t) \leq Z_{F}(t)$. Next, by setting $A=\left\{x \in F: d(x, C)<c_{4} t^{1 / d_{w}}\right\}$, where $c_{4}=\left(-\log \left(c_{3}\right) / c_{2}\right)^{1-1 / d_{w}}$, we have

$$
\begin{aligned}
Z_{F}(t)-\tilde{Z}_{F}(t) & \leq \int_{A} c_{5} t^{-d_{f} / d_{w}} \mu(d x)+\int_{F \backslash A} c_{6} t^{-d_{f} / d_{w}} e^{-c_{7}\left(\frac{d(x, C) d^{d_{w}}}{t}\right)^{1 /\left(d_{w}-1\right)}} \mu(d x) \\
& \leq c_{5} t^{-d_{f} / d_{w}} \mu(A)+c_{6} t^{-d_{f} / d_{w}} \int_{c_{4} t^{1 / d_{w}}}^{\infty} e^{-c_{7}\left(\epsilon^{d_{w}} t^{-1}\right)^{1 /\left(d_{w}-1\right)}} \nu(d \epsilon),
\end{aligned}
$$

where

$$
\nu(\epsilon)=\mu(\{x: d(x, C) \leq \epsilon\}) .
$$

We observe that from the construction of $C$ there is a constant $c$ such that $\nu(\epsilon) \leq c \epsilon^{d_{f}-d_{b_{1}}}$ and hence $\mu(A)=\nu\left(c_{4} t^{1 / d_{w}}\right) \leq c t^{\left(d_{f}-d_{b_{1}}\right) / d_{w}}$. Using these estimates we have

$$
Z_{F}(t) \leq \tilde{Z}_{F}(t)+c_{8} t^{-d_{b_{1}} / d_{w}},
$$

and hence

$$
\left|Z_{F}(t)-m_{F} Z_{F}\left(\tau_{F} t\right)\right| \leq c_{8} t^{-d_{b_{1}} / d_{w}}
$$

By setting $Z(t)=t^{-d_{f} / d_{w}} f(-\log t)$ we have

$$
\left|f(-\log t)-f\left(-\log t-\log \tau_{F}\right)\right| \leq c_{9} t^{\left(d_{f}-d_{b_{1}}\right) / d_{w}} .
$$

Thus putting $u=-\log t$ leads to a simple renewal equation

$$
f(u)=f\left(u-\log \tau_{F}\right)+g(u),
$$

where for $u>0$ we have

$$
|g(u)|=\left|f(u)-f\left(u-\log \tau_{F}\right)\right| \leq c_{10} e^{-u\left(d_{f}-d_{b_{1}}\right) / d_{w}} .
$$

For the case where $u<0$ we appeal to Lemma 3.7, where we see that for $u<0$ we have $t>1$ and for long times, by (3.3), we have exponential decay of the transition density and hence of the partition function,

$$
|g(u)|=\left|f(u)-f\left(u-\log \tau_{F}\right)\right| \leq c_{11} e^{-\lambda_{0} e^{-u}} .
$$

Thus we can apply our Lemma 3.5 to see that $f$ converges to a $\log \tau_{F}$-periodic function, with the appropriate error bound, and hence we have our result for the partition function.

Theorem 4.2 Let $F$ be a generalized Sierpinski carpet in $\mathbb{R}^{D}$. There exists a periodic function $\phi_{h}$ such that as $t \rightarrow 0$, the heat content satisfies

$$
E_{F}(t)=t^{\left(d_{f}-d_{b_{1}}\right) / d_{w}} \phi_{h}(-\log t)+O\left(t^{\left(d_{f}-d_{b_{2}}\right) / d_{w}}\right) .
$$

Proof: For the heat content we take a different decomposition of the carpet. Let $C$ be the $2(D-1)$ main diagonal hyperplanes for the unit hypercube. This divides the hypercube (and the carpet) up into $2 D$ hyperpyramids based on each face. We let $E_{1}(t)$ denote the heat content of one of these pieces, where the part of the boundary given by $C$ is set to have temperature 0 .

By construction $\tilde{E}_{F}(t)=2 D E_{1}(t)$ and applying Corollary 2.7 we have

$$
\left|E_{F}(t)-\tilde{E}_{F}(t)\right| \leq c_{1} \int_{\tilde{F}} \exp \left(-c_{2}\left(\frac{d(x, S)^{d_{w}}}{t}\right)^{1 /\left(d_{w}-1\right)}\right) \exp \left(-c_{3}\left(\frac{d(x, C)^{d_{w}}}{t}\right)^{1 /\left(d_{w}-1\right)}\right) \mu(d x) .
$$


We can estimate this integral by letting

$$
\nu(\epsilon)=\mu\left(\left\{x \in F: \frac{c_{2}}{p} d(x, S)^{d_{w} /\left(d_{w}-1\right)}+\frac{c_{3}}{q} d(x, C)^{d_{w} /\left(d_{w}-1\right)} \leq \epsilon^{d_{w} /\left(d_{w}-1\right)}\right\}\right) .
$$

Then we can write

$$
\begin{aligned}
\left|E_{F}(t)-\tilde{E}_{F}(t)\right| & \leq c_{1} \int_{\tilde{F}} \exp \left(-\frac{c_{2}}{p}\left(\frac{d(x, S)^{d_{w}}}{t}\right)^{1 /\left(d_{w}-1\right)}\right) \exp \left(-\frac{c_{3}}{q}\left(\frac{d(x, C)^{d_{w}}}{t}\right)^{1 /\left(d_{w}-1\right)}\right) \mu(d x) \\
& =c_{1} \int_{0}^{1} \exp \left(-\left(\frac{\epsilon^{d_{w}}}{t}\right)^{1 /\left(d_{w}-1\right)}\right) \nu(d \epsilon)
\end{aligned}
$$

Now we observe that

$$
\begin{array}{r}
\left\{x \in F: \frac{c_{2}}{p} d(x, S)^{d_{w} /\left(d_{w}-1\right)}+\frac{c_{3}}{q} d(x, C)^{d_{w} /\left(d_{w}-1\right)} \leq \epsilon^{d_{w} /\left(d_{w}-1\right)}\right\} \\
\left\{x \in F: d(x, S) \leq\left(\frac{p}{c_{2}}\right)^{1-1 / d_{w}} \epsilon, d(x, C) \leq\left(\frac{q}{c_{3}}\right)^{1-1 / d_{w}} \epsilon\right\}
\end{array}
$$

and let

$$
\left.\mu(\epsilon)=\mu\left(\left\{x \in F: d(x, S) \leq\left(\frac{p}{c_{2}}\right)^{1-1 / d_{w}} \epsilon, d(x, C) \leq\left(\frac{q}{c_{3}}\right)^{1-1 / d_{w}} \epsilon\right\}\right\}\right),
$$

so that $\nu(\epsilon) \leq \mu(\epsilon)$ for all $\epsilon$. As the intersection of $S$ and $C$ is contained in a $D-2$-dimensional hypersurface and has dimension $d_{b_{2}}$ it is straightforward to see that there is a constant $c_{4}$ such that $\mu(\epsilon) \leq c_{4} \epsilon^{d_{f}-d_{b_{2}}}$. Thus, using $\mu(\epsilon)$ and a suitable change of variable, we have

$$
\begin{aligned}
\left|E_{F}(t)-\tilde{E}_{F}(t)\right| & \leq c_{1} \int_{\tilde{F}} \exp \left(-\frac{c_{2}}{p}\left(\frac{d(x, S)^{d_{w}}}{t}\right)^{1 /\left(d_{w}-1\right)}\right) \exp \left(-\frac{c_{3}}{q}\left(\frac{d(x, C)^{d_{w}}}{t}\right)^{1 /\left(d_{w}-1\right)}\right) \mu(d x) \\
& \leq c_{5} \int_{0}^{1} \exp \left(-c_{6}\left(\frac{\epsilon^{d_{w}}}{t}\right)^{1 /\left(d_{w}-1\right)}\right) \mu(d \epsilon) \\
& \leq c_{7} \int_{0}^{1} \exp \left(-c_{6}\left(\frac{\epsilon^{d_{w}}}{t}\right)^{1 /\left(d_{w}-1\right)}\right) \epsilon^{d_{f}-d_{b_{2}}-1} d \epsilon \\
& \leq c_{7} t^{\left(d_{f}-d_{b_{2}}\right) / d_{w}} \int_{0}^{\infty} e^{-c_{6} u^{d_{w} /\left(d_{w}-1\right)}} u^{d_{f}-d_{b_{2}}-1} d u \\
& =c_{8} t^{\left(d_{f}-d_{b_{2}}\right) / d_{w}}
\end{aligned}
$$

Let $B$ denote the closed hyperpyramid in the carpet with outer boundary given by $C$ and the hyperplane $\left\{x_{1}=0\right\}$. We note that $F_{1} \cap B$ consists of $b_{1}$ cubes or parts of cubes of side $l_{F}^{-1}$ which intersect the boundary $\left\{x_{1}=0\right\}$ and some (possibly none) other cubes which do not. Thus $B$ can be decomposed into $B_{1}, \ldots, B_{b_{1}}$ which are scaled copies (by a factor $l_{F}^{-1}$ ) of the set $B$ and $B \backslash\left(B_{1} \cup \ldots \cup B_{b_{1}}\right)$ a set that has no intersection with the hyperplane $\left\{x_{1}=0\right\}$. For the two-dimensional carpet this decomposition is shown in Figure 1. We fix the temperature of the hyperplanes constituting the interior boundaries of the sets $B_{i}, i=1, \ldots, b_{1}$ to be 0 . This decomposes $B$ into $b_{1}+1$ regions, $b_{1}$ of which, $B_{1}, \ldots, B_{b_{1}}$, are scaled copies of $B$ with the same boundary conditions and the fourth region, $B^{\prime}=B \backslash\left(B_{1} \cup \ldots \cup B_{b_{1}}\right)$, which has 0 boundary conditions. We write $\tilde{E}_{B}$ for the heat content of the region $B$ with all the hyperplanes except the exterior held at temperature 0 and the exterior held at temperature 1 . Thus, as $B^{\prime}$ does not contribute to $\tilde{E}_{B}$, we have by $(3.2)$, that $\tilde{E}_{B}(t)=\frac{b_{1}}{m_{F}} E_{B}\left(\tau_{F} t\right)$. 
The same argument using the comparison lemma as for the whole carpet shows that there is a constant $c$ such that

$$
0 \leq E_{B}(t)-\tilde{E}_{B}(t) \leq c t^{\left(d_{f}-d_{b_{2}}\right) / d_{w}}
$$

and hence

$$
\left|E_{B}(t)-\frac{b_{1}}{m_{F}} E_{B}\left(\tau_{F} t\right)\right| \leq c t^{\left(d_{f}-d_{b_{2}}\right) / d_{w}}
$$

By taking the transformation $E_{B}(t)=t^{\left(d_{f}-d_{b_{1}}\right) / d_{w}} f(-\log t)$ we have

$$
\left|f(u)-f\left(u-\log \tau_{F}\right)\right| \leq c e^{-u\left(d_{b_{1}}-d_{b_{2}}\right) / d_{w}} .
$$

Now define $g(u)=f(u)-f\left(u-\log \tau_{F}\right)$ so that

$$
f(u)=f\left(u-\log \tau_{F}\right)+g(u),
$$

with $|g(u)| \leq c e^{-u\left(d_{b_{1}}-d_{b_{2}}\right) / d_{w}}$ for $u>0$. In the case of $u<0$ we apply our Lemma 3.7 to deduce that

$$
|g(u)|=\left|f(u)-f\left(u-\log \tau_{F}\right)\right| \leq c e^{u\left(d_{f}-d_{b_{1}}\right) / d_{w}} e^{-\lambda_{0} e^{-u}} .
$$

Thus we can apply Lemma 3.5 to show that asymptotically $f$ is a periodic function. Returning to the expression for the heat content we deduce that there is a $\log \tau_{F}$-periodic function $\phi$ such that

$$
E_{F}(t)=t^{\left(d_{f}-d_{b_{1}}\right) / d_{w}} \phi(-\log t)+O\left(t^{\left(d_{f}-d_{b_{2}}\right) / d_{w}}\right),
$$

completing the proof of our main theorem.

We remark that for the $D$-dimensional Sierpinski carpet (where we remove only the central hypercube), we have $d_{f}=\log \left(3^{D}-1\right) / \log 3$ and $d_{b_{1}}=D-1, d_{b_{2}}=D-2$. For the three dimensional Menger sponge $d_{f}=\log 20 / \log 3, d_{b_{1}}=\log 8 / \log 3, d_{b_{2}}=1$. An open problem is to determine precise two term asymptotic expansions. We would conjecture that, for the two dimensional Sierpinski carpet, we should have further periodic functions $\hat{\phi}_{p}, \hat{\phi}_{h}$ such that

$$
Z_{F}(t)=t^{-d_{f} / d_{w}} \phi_{p}(-\log t)+t^{-1 / d_{w}} \hat{\phi}_{p}(-\log t)+o(1)
$$

and

$$
E_{F}(t)=t^{\left(d_{f}-1\right) / d_{w}} \phi_{h}(-\log t)+t^{d_{f} / d_{w}} \hat{\phi}_{h}(-\log t)+o(1) .
$$

\subsection{The eigenvalue counting function}

We might hope that the partition function, the Laplace transform of the eigenvalue counting function, could be used to give us results on the counting function. Unfortunately it is not possible to invert the transform and obtain oscillatory asymptotics. However we can use a similar technique to get a result on the integrated counting function.

We introduce the eigenvalue counting function. The Dirichlet and Neumann eigenvalue problems for the Laplace operator on the carpet (interpreted as the generator of the self-similar diffusion with absorbing and reflecting boundary conditions) are:

(1) The Dirichlet case:

$$
\begin{aligned}
-\Delta u & =\lambda u, \text { in } F \\
u & =0, \text { on } \partial F .
\end{aligned}
$$


(2) The Neumann case

$$
\begin{aligned}
-\Delta u & =\lambda u, \text { in } F \\
d u & =0, \text { on } \partial F
\end{aligned}
$$

where $d u$ has to be interpreted as a normal derivative on the boundary.

We will reformulate these definitions for the Dirichlet form. Let $\mathcal{F}_{0}=\{f: f \in \mathcal{F}, f(x)=$ $0, x \in \partial F\}$ and write $\mathcal{E}_{0}(f, f)=\mathcal{E}(f, f)$ for $f \in \mathcal{F}_{0}$ and $(.,$.$) for the inner product on L^{2}(F, \mu)$. Then we define $\lambda$ to be a Dirichlet eigenvalue with eigenfunction $u$ if

$$
\mathcal{E}_{0}(u, v)=\lambda(u, v), \quad \forall v \in \mathcal{F}_{0} .
$$

Similarly, $\lambda$ is defined to be a Neumann eigenvalue with eigenfunction $u$ if

$$
\mathcal{E}(u, v)=\lambda(u, v), \quad \forall v \in \mathcal{F} .
$$

By [5] Proposition 6.15 the Dirichlet Laplacian has a discrete spectrum. We write the spectrum as an increasing sequence of eigenvalues $0<\lambda_{1} \leq \lambda_{2} \leq \ldots$ with $\lambda_{n} \rightarrow \infty$ as $n \rightarrow$ $\infty$. Thus we can define the Dirichlet eigenvalue counting function to be $N_{0}(x)=\max \{i$ : $\lambda_{i} \leq x, \lambda_{i}$ is a Dirichlet eigenvalue $\}$. Similarly there is a discrete spectrum for the Neumann Laplacian and we define the Neumann eigenvalue counting function $N(x)=\max \left\{i: \lambda_{i} \leq\right.$ $x, \lambda_{i}$ is a Neumann eigenvalue $\}$.

In order to deduce properties of the counting functions we use Dirichlet-Neumann bracketing. We write $F_{1}$ for the union of the hyperplanes which divide the carpet into its level 1 hypercubes. Let $(\tilde{\mathcal{E}}, \tilde{\mathcal{F}})$ be defined by

$$
\tilde{\mathcal{F}}=\left\{f: F \backslash F_{1} \rightarrow \mathbb{R}, f \circ \psi_{i}=f_{i} \text { on } F \backslash \partial F, \text { for some } f_{i} \in \mathcal{F}\right\},
$$

and

$$
\tilde{\mathcal{E}}(f, g)=\sum_{i=1}^{m_{F}} \rho_{F} \mathcal{E}\left(f \circ \psi_{i}, g \circ \psi_{i}\right), \quad f, g \in \tilde{\mathcal{F}} .
$$

Also let $\left(\tilde{\mathcal{E}}_{0}, \tilde{\mathcal{F}}_{0}\right)$ be defined by

$$
\tilde{\mathcal{F}}_{0}=\left\{f: f \in \mathcal{F}_{0},\left.f\right|_{F_{1}}=0\right\},
$$

and

$$
\tilde{\mathcal{E}}_{0}(f, g)=\sum_{i=1}^{m_{F}} \mathcal{E}_{0}\left(f \circ \psi_{i}, g \circ \psi_{i}\right), \quad f, g \in \tilde{\mathcal{F}}_{0} .
$$

It is straightforward to see that

$$
\tilde{\mathcal{F}}_{0} \subset \mathcal{F}_{0} \subset \mathcal{F} \subset \tilde{\mathcal{F}} .
$$

We also note that $(\tilde{\mathcal{E}}, \tilde{\mathcal{F}})$ and $\left(\tilde{\mathcal{E}}_{0}, \tilde{\mathcal{F}}_{0}\right)$ are local regular Dirichlet forms on $L^{2}(F, \mu)$ and they have discrete spectra. The key observation is that if $\lambda$ is a Neumann eigenvalue, with eigenfunction $u$, then for all $v \in \mathcal{F}$

$$
\sum_{i=1}^{m_{F}} \rho_{F} \mathcal{E}\left(u \circ \psi_{i}, v \circ \psi_{i}\right)=\mathcal{E}(u, v)=\lambda(u, v)=\lambda \sum_{i=1}^{m_{F}} \frac{1}{m_{F}}\left(u \circ \psi_{i}, v \circ \psi_{i}\right) .
$$


If we take $v$ to be a function supported on a 1-cell with address $i$, we see that

$$
\mathcal{E}\left(u \circ \psi_{i}, v \circ \psi_{i}\right)=\frac{\lambda}{\rho_{F} m_{F}}\left(u \circ \psi_{i}, v \circ \psi_{i}\right),
$$

thus $\lambda / \tau_{F}$ is also an eigenvalue with eigenfunction $u_{i}=u \circ \psi_{i}$. From this we can construct eigenfunctions and eigenvalues for $(\tilde{\mathcal{E}}, \tilde{\mathcal{F}})$, as

$$
\tilde{u}(x)= \begin{cases}u_{i}(x), & x \in F \cap \operatorname{int}\left(\psi_{i}(F)\right), \\ 0, & x \notin F \cap \operatorname{int}\left(\psi_{i}(F)\right),\end{cases}
$$

gives an eigenfunction with eigenvalue $\lambda$. Hence

$$
\tilde{N}(x)=\sum_{i=1}^{m_{F}} N\left(x / \tau_{F}\right)=m_{F} N\left(x / \tau_{F}\right) .
$$

Similarly we can show that

$$
\tilde{N}_{0}(x)=\sum_{i=1}^{m_{F}} N_{0}\left(x / \tau_{F}\right)=m_{F} N_{0}\left(x / \tau_{F}\right)
$$

Lemma 4.3 The following relationships hold

$$
m_{F} N_{0}\left(x / \tau_{F}\right) \leq N_{0}(x) \leq N(x) \leq m_{F} N\left(x / \tau_{F}\right),
$$

and

$$
m_{F} \bar{N}_{0}\left(x / \tau_{F}\right) \leq \bar{N}_{0}(x) \leq \bar{N}(x) \leq m_{F} \bar{N}\left(x / \tau_{F}\right) .
$$

In order to apply Dirichlet-Neumann bracketing all that is needed is to establish a comparison between the Dirichlet and Neumann counting functions. Unfortunately we do not have quite enough information about $N(x)-N_{0}(x)$ to get a result on the counting function itself. Instead we can deduce oscillation for the integrated counting function $\bar{N}(x)=\int_{0}^{x} N(y) d y$. To do this we use the comparison lemma for the transition density.

Lemma 4.4 There exists a constant $c$ such that

$$
\bar{N}_{0}(x) \leq \bar{N}(x) \leq \bar{N}_{0}(x)+c x^{1+d_{b_{1}} / d_{w}} .
$$

Proof: The left inequality is trivial. For the right we use the fact that we can write

$$
\int_{0}^{\infty} t e^{-s t} N(s) d s=\int_{F} p_{t}(x, x) \mu(d x),
$$

and from the relationships between transforms

$$
\int_{0}^{\infty} t e^{-s t} \bar{N}(s) d s=\frac{1}{t} \int_{F} p_{t}(x, x) \mu(d x) .
$$

Applying the comparison result in Remark 2.5 we have,

$$
\begin{aligned}
\int_{0}^{\infty} t e^{-s t}\left(\bar{N}(s)-\bar{N}_{0}(s)\right) d s & =\frac{1}{t} \int_{F}\left(p_{t}(x, x)-p_{t}^{a}(x, x)\right) \mu(d x), \\
& =\frac{1}{t} \int_{A}\left(p_{t}(x, x)-p_{t}^{a}(x, x)\right) \mu(d x)+\frac{1}{t} \int_{F \backslash A}\left(p_{t}(x, x)-p_{t}^{a}(x, x)\right) \mu(d x)
\end{aligned}
$$


where $A=\left\{x: d(x, \partial F) \leq\left(-\log c_{3} / c_{2}\right)^{1-1 / d_{w}} t^{1 / d_{w}}\right\}$ (as we can take $c_{3}<1$ in Remark 2.5). Now we follow the same argument as in the proof of Theorem 4.1 from (4.1) to obtain

$$
\int_{0}^{\infty} t e^{-s t}\left(\bar{N}(s)-\bar{N}_{0}(s)\right) d s \leq c t^{-1-d_{b_{1}} / d_{w}} .
$$

As the function $\bar{N}(x)-\bar{N}_{0}(x)=\int_{0}^{x}\left(N(y)-N_{0}(y)\right) d y$ is monotone increasing we have for all $t>0$,

$$
\begin{aligned}
c t^{-1-d_{b} / d_{w}} & \geq \int_{0}^{\infty} t e^{-s t}\left(\bar{N}(s)-\bar{N}_{0}(s)\right) d s \\
& \geq \int_{x}^{2 x} t e^{-s t}\left(\bar{N}(s)-\bar{N}_{0}(s)\right) d s \\
& \geq x t e^{-2 x t}\left(\bar{N}(x)-\bar{N}_{0}(x)\right) .
\end{aligned}
$$

Now choose $t=1 / x$ to see that

$$
\bar{N}(x)-\bar{N}_{0}(x) \leq c x^{1+d_{b_{1}} / d_{w}},
$$

as desired.

We are now ready to prove a result for the integrated eigenvalue counting function.

Theorem 4.5 There exist periodic functions $\phi_{0}(x), \phi(x)$ such that as $x \rightarrow \infty$,

$$
\bar{N}_{0}(x)=x^{1+d_{f} / d_{w}} \phi_{0}(\log x)+O\left(x^{1+d_{b_{1}} / d_{w}}\right),
$$

and

$$
\bar{N}(x)=x^{1+d_{f} / d_{w}} \phi(\log x)+O\left(x^{1+d_{b_{1}} / d_{w}}\right) .
$$

Proof: The proof is a reduction to the renewal equation. Let $\bar{N}_{0}(x)=x^{1+d_{f} / d_{w}} f(\log x)$ and write $g(\log x)=x^{-\left(1+d_{f} / d_{w}\right)}\left(\bar{N}_{0}(x)-m_{F} \bar{N}_{0}\left(x / \tau_{F}\right)\right)$ to see that

$$
f(u)=f\left(u-\log \tau_{F}\right)+g(u),
$$

where $\left.g(u) \leq c \exp \left(-u\left(d_{f}-d_{b_{1}}\right) / d_{w}\right)\right)$ for $u$ large. For $u<0$ we use the existence of a strictly positive bottom eigenvalue to see that $g(u)=0$ for $u<u_{*}$. An application of the renewal theorem as given in Lemma 3.5 gives the result.

The result for $\bar{N}(x)$ follows using the upper estimate in Lemma 4.4, which shows that the difference between the two functions is $O\left(x^{1+d_{b_{1}} / d_{w}}\right)$ as $x \rightarrow \infty$.

\subsection{The on-diagonal transition density}

Finally we consider the on-diagonal transition density and we show how oscillations occur in this function at particular points. Let $x_{\xi} \notin \partial F$ be such that it is a fixed point for a finite combination of the similitudes that are used to construct the fractal. Thus there is a map $\xi=\psi_{i_{1}} \circ \ldots \circ \psi_{i_{k}}$ such that $x_{\xi}=\xi\left(x_{\xi}\right)$. We assume that $\xi$ is the minimal map (in the sense of number of compositions of similitudes) which fixes $x_{\xi}$. Once again we will use our comparison theorem to derive a renewal equation to obtain oscillatory asymptotics. 
By the scaling, we have for the map $\xi$, that

$$
p_{t}^{a}(x, x)=m_{F}^{-k} p_{\tau_{F}^{-k} t}^{a, \xi(\partial F)}(\xi(x), \xi(x)), \quad \forall x \in F .
$$

The comparison theorem allows us to compare the transition density with absorption on the boundary of the smaller copy with the transition density with absorption on the original boundary. Thus for any $x \in F$ and map $\xi$ there are constants $c, \tilde{c}$ such that

$$
h(t, x):=p_{t}^{a}(\xi(x), \xi(x))-p_{t}^{a, \xi(\partial F)}(\xi(x), \xi(x)) \leq c t^{-d_{f} / d_{w}} \exp \left(-\tilde{c}\left(d(\xi(x), \xi(\partial F))^{d_{w}} / t\right)^{1 /\left(d_{w}-1\right)}\right) .
$$

By scaling we have $d(\xi(x), \xi(\partial F))=l_{F}^{-k} d(x, \partial F)$ and hence

$$
h(t, x) \leq c t^{-d_{f} / d_{w}} \exp \left(-\tilde{c}\left(d(x, \partial F)^{d_{w}} / \tau^{k} t\right)^{1 /\left(d_{w}-1\right)}\right) .
$$

Now consider the fixed point $x_{\xi}$ and let $p_{t}^{a}\left(x_{\xi}, x_{\xi}\right)=t^{-d_{f} / d_{w}} f(\log 1 / t)$. As

$$
p_{t}^{a}\left(x_{\xi}, x_{\xi}\right)=m_{F}^{-k} p_{\tau_{F}^{-k} t}^{a}\left(x_{\xi}, x_{\xi}\right)-m_{F}^{-k} h\left(\tau_{F}^{-k} t, x_{\xi}\right),
$$

we have, by setting $u=-\log t$, a renewal equation

$$
f(u)=f\left(u-k \log \tau_{F}\right)+g(u),
$$

where $g(\log 1 / t)=-m_{F}^{-k}\left(\tau_{F}^{-k} t\right)^{d_{f} / d_{w}} h\left(\tau_{F}^{-k} t, x_{\xi}\right)$. By (4.2) we have that

$$
\begin{aligned}
-g(-\log t) & \leq c m_{F}^{-k}\left(\tau_{F}^{-k} t\right)^{d_{f} / d_{w}}\left(\tau_{F}^{-k} t\right)^{-d_{f} / d_{w}} \exp \left(-\tilde{c} t^{-1 /\left(d_{w}-1\right)}\right) \\
& =c m_{F}^{-k} \exp \left(-\tilde{c} t^{-1 /\left(d_{w}-1\right)}\right) .
\end{aligned}
$$

Thus there is a constant $c^{\prime}(k)$ such that $|g(u)| \leq c^{\prime}(k) \exp \left(-\tilde{c} e^{u /\left(d_{w}-1\right)}\right)$ for $u>0$ For $u<0$ we have by Lemma 3.7 that $|g(u)| \leq c_{0} \exp \left(-\lambda_{0} e^{-u}\right)$. An application of Remark 3.6 following our renewal theorem gives the following result.

Theorem 4.6 For any point $x$, a fixed point of a composition of $k$ maps, such that $x \notin \partial F$ we have a periodic function $\phi$ of period $k \log \tau_{F}$ such that as $t \rightarrow 0$,

$$
p_{t}^{a}(x, x)=t^{-d_{f} / d_{w}} \phi(-\log t)+O\left(e^{-c t^{-1 /\left(d_{w}-1\right)}}\right) .
$$

Remark 4.7 This result shows that non-boundary points with periodic addresses show asymptotic oscillations for their transition density. This set forms a subset of the fractal with Hausdorff measure 0 . As there is asymptotic oscillation in the partition function, the integral of the transition density, we would anticipate that there is asymptotic oscillation on a set of points of positive measure.

\section{$5 \quad$ Affine nested fractals}

We have dealt initially with the Sierpinski carpet but the comparison lemmas of Section 2 can be used to derive some results about finitely ramified fractals as well. We will mainly discuss the heat content as the eigenvalue counting function asymptotics are well known in this setting [21] and, by inversion of the Laplace transform, lead immediately to the partition function results. We also note that the on-diagonal transition density result for carpets was derived in a way which was 
essentially independent of the set, just relying on the comparison lemma and the scaling. Thus we can state a version of Theorem 4.6 for affine nested fractals as well.

For the heat content on the Sierpinski gasket there are two natural boundaries that could be used. As in the carpet case we can consider the embedding of the fractal in $\mathbb{R}^{2}$. The other possibility is to use the natural intrinsic boundary of the fractal - the three vertices of the outer triangle. We will see that sharper asymptotics can be obtained in this case.

We will not give the full definitions here, they can be found in $[1,14]$, but note that affine nested fractals are nested fractals which may have different contraction factors (which respect the symmetries of the nested fractal). It is now well known that nested and affine nested fractals support a $\operatorname{FD}\left(d_{f}, d_{w}\right)$, through the work of $[27,22,14]$, where the fractional metric space is $(F, d)$, where $d$ is a shortest path metric.

An affine nested fractal $F$ is constructed from a family $\left\{\psi_{i}, i=1, . ., N\right\}$ of similitudes. We label the 1-cells using a type, one for each of the sets of 1-cells which are invariant under the symmetries of the set. We allocate a resistance weight $\rho_{i}$ to a 1-cell of a given type $i$. Assume that there are $M$ types and $N_{i}$ 1-cells of type $i$. We also assume that each boundary point is only contained in one 1-cell. We recall that the natural measure for an affine nested fractal is the Bernoulli measure in which a 1-cell of type $i$ has $\mu_{i}=\rho_{i}^{-S}$, where $S$ is defined to be such that $\sum_{i=1}^{M} N_{i} \rho_{i}^{-S}=1$. Then the timescale factors $\tau_{i}=\rho_{i} / \mu_{i}=\rho_{i}^{1+S}$. We note that the Hausdorff dimension and walk dimension of the fractal in the resistance metric are given by $d_{f}^{r}=S$ and $d_{w}^{r}=S+1$ and thus the spectral dimension is $d_{s}=2 S /(S+1)$ (which is independent of the metric). Otherwise the exponents $d_{f}, d_{w}$ are with respect to the shortest path metric.

Theorem 5.1 (a) There exists a local regular Dirichlet form $(\mathcal{E}, \mathcal{F})$ on $L^{2}(F, \mu)$ with the selfsimilar decomposition

$$
\mathcal{E}(f, g)=\sum_{i=1}^{N} \rho_{i} \mathcal{E}\left(f \circ \psi_{i}, g \circ \psi_{i}\right) .
$$

(b) The corresponding stochastic process $\left\{X_{t}: t \geq 0\right\}$ on $(\Omega, \mathcal{F}, P)$ with state space $F$ satisfies the scaling law that for any similitude $\psi_{i}$, for $A \subset F, x \in A$, the law of $\left\{\psi_{i}\left(X_{t}\right): 0 \leq t \leq T_{A}\right\}$ under $P^{x}$ is equal to the law of $\left\{X_{t / \tau_{i}}: 0 \leq t \leq T_{\psi_{i}(A)}\right\}$ under $P^{\psi_{i}(x)}$.

(c) There exists a jointly continuous transition density $p_{t}(x, y)$ with respect to the natural measure which satisfies the sub-Gaussian bounds, for all $x, y \in F$ and $t \geq 0$, there are constants such that

$$
c_{1} t^{-d_{f} / d_{w}} \exp \left(-c_{2}\left(\frac{d(x, y)^{d_{w}}}{t}\right)^{1 /\left(d_{w}-1\right)}\right) \leq p_{t}(x, y) \leq c_{3} t^{-d_{f} / d_{w}} \exp \left(-c_{4}\left(\frac{d(x, y)^{d_{w}}}{t}\right)^{1 /\left(d_{w}-1\right)}\right) .
$$

(d) There exists a jointly continuous transition density $p_{t}^{a, C}(x, y)$ with respect to the natural measure for the process $X$ with absorption on $\partial F \cup C$ for any closed set $C$.

Proof: (a), (b) and (c) are standard, see [14].

(d) This is essentially shown for the Sierpinski gasket in [9] Theorem 7.11(a). The case of more general affine nested fractals can be established using the approach for the carpet as given in [5].

The Sierpinski gasket $G$ supports a $\mathrm{FD}(\log 3 / \log 2, \log 5 / \log 2)$ as a fractional metric space $(G,||$.$) with the Euclidean metric.$ 


\subsection{The partition function and transition density}

The partition function result is the exact analogue of the original result for the eigenvalue counting function obtained in [21] for p.c.f. self-similar sets and could be derived for p.c.f. self-similar sets by integrating the result on the eigenvalue counting function derived in [21].

Theorem 5.2 For any affine nested fractal:

1. If $\sum_{i=1}^{N} \mathbb{Z} \log N \rho_{i}$ is a dense subgroup of $\mathbb{R}$, then there exists a constant $c_{1}$ such that

$$
\lim _{t \rightarrow 0} t^{d_{s} / 2} Z_{F}(t)=c_{1} .
$$

2. If $\sum_{i=1}^{N} \mathbb{Z} \log N \rho_{i}$ is a discrete subgroup of $\mathbb{R}$, then if $T$ is its generator, there is a positive $T$-periodic function $\phi$ such that as $t \rightarrow 0$,

$$
Z_{F}(t)=t^{-d_{s} / 2}(\phi(-\log t)+o(1)) .
$$

3. If $\rho_{i}=\rho$ for all $i=1, \ldots, N$, then there is a $\log \tau$-periodic function $\phi(t)$ such that as $t \rightarrow 0$,

$$
Z_{F}(t)=t^{-d_{s} / 2} \phi(-\log t)+O(1)
$$

For the on-diagonal transition density we observe that the same argument as for the Sierpinski carpet used in Theorem 4.6 can be applied.

Theorem 5.3 For any point $x$, a fixed point of a composition of $k$ maps, $\psi_{i_{j}}, j=1, \ldots, k, i_{j} \in$ $\{1, \ldots, N\}\}$, such that $x \notin \partial F$ we have a periodic function $\phi$ of period $(S+1) \sum_{j=1}^{k} \log \rho_{i_{j}}$ and a constant $c$ such that as $t \rightarrow 0$,

$$
p_{t}^{a}(x, x)=t^{-d_{s} / 2} \phi(-\log t)+O\left(e^{-c t^{-1 /\left(d_{w}-1\right)}}\right) .
$$

\subsection{The heat content}

We now turn to the heat content where the results are a little different depending on the embedding of the fractal into $\mathbb{R}^{D}$. We remark that the scaling result

$$
E_{\psi_{i}(F)}(t)=\rho_{i}^{-S} E_{F}\left(\tau_{i} t\right)
$$

will hold for affine nested fractals.

For the first case we take $\partial F=V_{0}$ and we regard the fractal as a self-sufficient metric space with boundary $V_{0}$. The computation of the heat content is very straightforward. We let $C$ be the vertices of $V_{1} \backslash V_{0}$ and set the temperature at these points to be 0 . We write $\tilde{E}_{F}(t)$ for the associated heat content.

We now apply the comparison Lemma 2.6. Note that in this case as there is no intersection between the sets $C$ and $S$ we have a much sharper estimate in that

$$
\left|E_{F}(t)-\tilde{E}_{F}(t)\right| \leq c_{1} e^{-c_{2} t^{-1 /\left(d_{w}-1\right)}} .
$$

We can now apply scaling to each of the $N$ copies that make up $\tilde{F}$. We note that all but those copies containing a vertex of $V_{0}$ will have zero boundary conditions and thus will not contribute to $\tilde{E}_{F}(t)$. Also those copies attached to a vertex in $V_{0}$ must have the same scale factor by the symmetry assumptions for an affine nested fractal, without loss of generality we label the resistance 
weight of these copies $\rho_{1}$. Thus $E_{\tilde{F}}(t)=\left|V_{0}\right| E_{1}(t)$, where $E_{1}(t)$ is without loss of generality the heat content of the copy $\psi_{1}(F)$ where one boundary point is held at temperature 1, and the rest at temperature 0. Then, $E_{1}(t)=\sum_{j=1}^{N} \tilde{E}_{1 j}(t)$, where the $\tilde{E}_{i j}=\tilde{E}_{\psi_{1} \circ \psi_{j}(F)}$ are the heat contents of the components of $\psi_{1}(F)$. As all but one of these has 0 boundary conditions and the one that does not satisfies $\tilde{E}_{1 j}=\rho_{1}^{-S} E_{1}\left(\rho_{1}^{1+S} t\right)$ by scaling, we have, with another application of the comparison lemma,

$$
E_{1}(t)-\rho_{1}^{-S} E_{1}\left(\rho_{1}^{1+S} t\right) \leq c_{3} e^{-c_{4} t^{-1 /\left(d_{w}-1\right)}} .
$$

From this observation the following theorem is straightforward, using Remark 3.6.

Theorem 5.4 For an affine nested fractal with outer boundary $V_{0}$ there exists a periodic function, $\phi_{2}$ with period $(1+S) \log \rho_{1}$, such that as $t \rightarrow 0$,

$$
E_{F}(t)=t^{d_{f} / d_{w}} \phi_{2}(-\log t)+O\left(e^{-c t^{-1 /\left(d_{w}-1\right)}}\right) .
$$

Next we consider affine nested fractals embedded in $\mathbb{R}^{2}$. In order to indicate the approach we briefly discuss the Sierpinski gasket before giving the general result. For this fractal the boundary is the unit triangle and, as before, we decompose the fractal into pieces, then use the comparison lemma to obtain a renewal equation. In this setting we have to be a little more careful as the behaviour of the different pieces is slightly different. It is clear that we can divide the original triangle up into three level one pieces each with two sides held at temperature 1 as in the top line of Figure 5.2. The next stage is to divide a level one triangle up into three pieces, one of which has two edges at temperature 1 , the other two having one edge at temperature 1 . Let $E_{i}(t)$ be

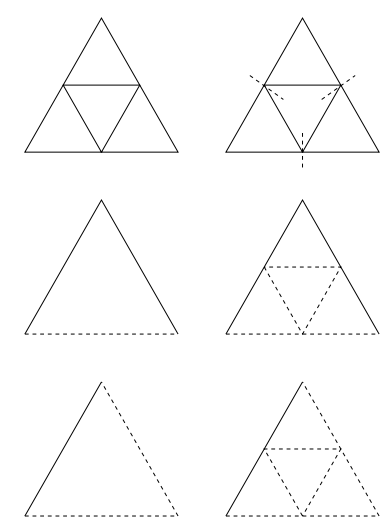

Figure 2: decomposition for the Sierpinski gasket with boundary the unit triangle

the heat content for a triangle with $i$ sides held at temperature 1 . We write $\tilde{E}_{i}(t)$ for the heat content of a triangle with $i$ sides held at temperature 1 and its internal connection points held at temperature 0. By considering how these triangles break down, as shown in the bottom two lines of Figure 5.2, we can obtain a two dimensional recurrence

$$
\begin{aligned}
\tilde{E}_{1}(t) & =\frac{2}{3} E_{1}(5 t), \\
\tilde{E}_{2}(t) & =\frac{1}{3} E_{2}(5 t)+\frac{2}{3} E_{1}(5 t)
\end{aligned}
$$

where $\left|\tilde{E}_{i}(t)-E_{i}(t)\right| \leq c_{i} t^{d_{f} / d_{w}}$. A simple renewal analysis of the first component $E_{1}(t)$, setting $E_{1}(t)=t^{\left(d_{f}-1\right) / d_{w}} f_{1}(-\log t)$, gives the existence of a periodic function $\phi_{1}$ such that $E_{1}(t)=$ 
$t^{\left.\left(d_{f}-1\right) / d_{w}\right)} \phi_{1}(-\log t)+O\left(t^{d_{f} / d_{w}}\right)$ as $t \rightarrow 0$. As we can write $\tilde{E}_{2}(t)=\tilde{E}_{1}(t)+\frac{1}{3} E_{2}(5 t)$, we have

$$
E_{2}(t)=E_{1}(t)+\frac{1}{3} E_{2}(5 t)+h(t),
$$

where $h(t)=\left|\tilde{E}_{1}(t)-E_{1}(t)+E_{2}(t)-\tilde{E}_{2}(t)\right| \leq\left(c_{1}+c_{2}\right) t^{d_{f} / d_{w}}$ for $t \leq 1$. For $t>1$, by the analogue of Lemma 3.7, we have $h(t) \leq 2 c_{0} e^{-\lambda_{0} t}$. Now we have, by setting $E_{2}(t)=t^{\left(d_{f}-1\right) / d_{w}} f_{2}(-\log t)$, that

$$
f_{2}(u)=\frac{1}{2} f_{2}(u-\log 5)+f_{1}(u)+g(u) .
$$

We note that $g(u)=e^{\left(d_{f}-1\right) u / d_{w}} h\left(e^{-u}\right) \leq c e^{-u / d_{w}}$ for $u \geq 0$, while for $u<0$ we have $g(u) \leq$ $2 c_{0} e^{-\lambda_{0} e^{-u}}$. As $f_{1}(u)$ converges to a $\log 5$ periodic function we see that the difference between $f_{2}(u)$ and this function is also a $\log 5$ periodic function and hence we have the following result.

Theorem 5.5 For the Sierpinski gasket with outer boundary consisting of the unit triangle in $\mathbb{R}^{2}$, there exists a periodic function, $\phi_{3}(\log t)$ with period $\log 5$, such that

$$
E_{G}(t)=t^{\left(d_{f}-1\right) / d_{w}} \phi_{3}(\log t)+O\left(t^{d_{f} / d_{w}}\right) .
$$

We will now give a theorem for a subclass of affine nested fractals. Firstly we recall that, as remarked in [1], the symmetry assumptions for nested fractals restrict the initial set to be a regular $L$-sided polygon in $\mathbb{R}^{2}$ or a $D$-dimensional tetrahedron or a $D$-dimensional simplex. We will only consider the polygons in $\mathbb{R}^{2}$ and write $L$ for the number of sides of our fractal. We let $d_{b}$ denote the dimension of the boundary. In the resistance metric this is $S_{b}$ such that $\sum_{i=1}^{N_{b}} \rho_{i}^{S_{b}}=1$, where $N_{b}$ is the number of cells that have an open subset of their boundaries on a side of the fractal.

Theorem 5.6 Let $F$ be an affine nested fractal in $\mathbb{R}^{2}$. If $\sum_{i} \log \rho_{i} \mathbb{Z}$ is a discrete subgroup of $\mathbb{R}$, then there exists a periodic function $\phi$ such that

$$
E_{F}(t)=t^{\left(d_{f}-d_{b}\right) / d_{w}}(\phi(-\log t)+o(1)) .
$$

If $\sum_{i} \log \rho_{i} \mathbb{Z}$ is a dense subgroup of $\mathbb{R}$, then there exists a constant $K$ such that

$$
E_{F}(t)=t^{\left(d_{f}-d_{b}\right) / d_{w}}(K+o(1)) .
$$

Proof: We label the $N$ level one cells of $F$ as $B_{j}^{(k)}, j=1, \ldots, n_{k}, k=0, \ldots, L$, where we classify them according to the number of sides $k$ of these cells which are in the boundary $\partial F$. We call a set $B_{j}^{(k)}$ a side-type $k$ cell and $n_{k}$ is the number of such cells. Thus

$$
F=\bigcup_{k=0}^{L} \bigcup_{j=1}^{n_{k}} B_{j}^{k},
$$

and

$$
\partial F=\partial \bigcup_{k=1}^{L} \bigcup_{j=1}^{n_{k}} B_{j}^{k} .
$$

The set $C$ is chosen to be $\left(\bigcup_{k=0}^{L} \bigcup_{j=1}^{n_{k}} \partial B_{j}^{k}\right) \backslash \partial F$, that is the edges in the boundaries of all the one cells which do not lie in the outer boundary $\partial F$ of the fractal. 
Hence, writing $\tilde{E}_{F}$ for the heat content of the set $F$ with the set $C$ held at temperature 0,

$$
\tilde{E}_{F}(t)=\sum_{k=1}^{L} \sum_{j=1}^{n_{k}} E_{B_{j}^{(k)}}(t)
$$

Applying the comparison lemma we obtain for $t<1$,

$$
\left|E_{F}(t)-\tilde{E}_{F}(t)\right| \leq c t^{d_{f} / d_{w}} .
$$

For $t>1$ we observe that the analogue of (3.4) holds for nested fractals and hence for $t>1$, there are constants $c_{0}, \lambda_{0}$ such that

$$
\left|E_{F}(t)-\tilde{E}_{F}(t)\right| \leq c_{0} \exp \left(-\lambda_{0} t\right) .
$$

By symmetry we just need to consider the heat content $E_{i}(t)$ for one of the side-type $i$ cells $B^{(i)}$ where the edges of $B^{(i)} \cap C$ are held at temperature 0 and the edges of $B^{(i)} \cap \partial F$ held at temperature 1 . If we write $n_{i j k}$ for the number of side-type $j$ cells in a side-type $i$ cell of type $k$ (and hence with resistance weight $\rho_{k}$ ), then by scaling and symmetry

$$
\tilde{E}_{B^{(i)}}(t)=\sum_{j=1}^{L} \sum_{k=1}^{M} n_{i j k} \rho_{k}^{-S} E_{j}\left(\tau_{k} t\right)
$$

where $\tilde{E}_{B^{(i)}}$ is the heat content of the set $B^{(i)}$ with the edges of the scaled copy of $C$ inside it held at temperature 0 . Using our comparison lemma in the same way as before we have

$$
E_{i}(t)=\sum_{j=1}^{L} \sum_{k=1}^{M} n_{i j k} \rho_{k}^{-S} E_{j}\left(\tau_{k} t\right)+R_{i}(t)
$$

where $R_{i}(t) \leq c t^{d_{f} / d_{w}}$.

Now, as $\left(d_{f}-d_{b}\right) / d_{w}=\left(S-S_{b}\right) /(S+1)$, we let $E_{i}(t)=t^{\left(d_{f}-d_{b}\right) / d_{w}} f_{i}(-\log t)$ to get the renewal equation

$$
\begin{aligned}
f_{i}(-\log t) & =\sum_{j=1}^{L} \sum_{k=1}^{M} n_{i j k} \rho_{k}^{-S} \tau_{k}^{\left(d_{f}-d_{b}\right) / d_{w}} f_{j}\left(-\log t-\log \tau_{k}\right)+t^{\left(d_{b}-d_{f}\right) / d_{w}} R_{i}(t) \\
f_{i}(u) & =\sum_{j=1}^{L} \sum_{k=1}^{M} n_{i j k} \rho_{k}^{-S_{b}} f_{j}\left(u-\log \tau_{k}\right)+g_{i}(u),
\end{aligned}
$$

where $\left|g_{i}(u)\right| \leq e^{-|u| d_{b} / d_{w}}$.

We note that the boundary of an affine nested fractal is a self-similar set and the boundary dimension can be computed in the resistance metric by standard techniques for such graph directed sets [28]. Thus if $A(s)$ is the matrix with $A_{i j}(s)=\sum_{k=1}^{M} n_{i j k} \rho_{k}^{-s}$, then $S_{b}$ is such that the maximum eigenvalue of $A\left(S_{b}\right)=1$. Thus we have a multidimensional renewal equation

$$
f(u)=F \star f(u)+g(u),
$$

where $F(t)$ is the matrix of distribution functions $F_{i j}(t)=\sum_{k} n_{i j k} \rho_{k}^{-S_{b}} I\left(\tau_{k}<t\right)$. By construction $F(\infty)$ has maximum eigenvalue 1 and is irreducible and therefore by the multidimensional renewal theorem of [25] we have $f(u) \rightarrow c$ as $u \rightarrow \infty$ under a non-lattice condition. It is easily seen that 
this non-lattice condition is equivalent to the stated condition on $\log \rho_{k}$. Similarly in the lattice case we have the existence of a periodic function.

We have already seen how the Sierpinski gasket can be treated. The Lindstrøm snow flake is another example. This is a hexagonal fractal and thus has 6 sides. It is easy to see that the boundary cells have 4 sides intersecting the boundary and that these 4 sided cells split into two 2 sided cells and three 4 sided cells. Similarly the 2 sided cells split into two 2 sided cells and one 4 sided cell and a straightforward computation gives the boundary dimension when we assume that $\rho_{k}=1$ for $k=1, \ldots, 7$. Thus it is easy to apply our theorem with $d_{f}=\log 7 / \log 3, d_{b}=\log 4 / \log 3$ and $d_{w}=\log \tau / \log 3$. It is clearly lattice and hence we have a periodic function in the limit.

We note that the Lindstrøm snowflake has an inverted triangular Koch curve as a boundary. In the case of the snowflake domain in $\mathbb{R}^{D}$ there is a second order term for the heat content that is periodic with period $\log 9$. It would be interesting to determine the second order term for this fractal. We would conjecture that, as in the snowflake domain in $\mathbb{R}^{2}$, there will be a second order term of the form $t^{d_{f} / d_{w}} \phi(-\log t)$ where $\phi$ is a periodic function of period $\log \tau$.

Finally, in the case of the affine nested fractal considered in [23] and shown in Figure 5.2, we can show an example of constant behaviour for the heat content. The unit triangle is decomposed into seven triangles as shown. The triangles labelled $U$ are given resistance scale factor $\rho_{1}$, the triangles labelled $V$ have resistance scale factor $\rho_{2}$ and the triangle labelled $W$ has resistance scale factor $\rho_{3}$.

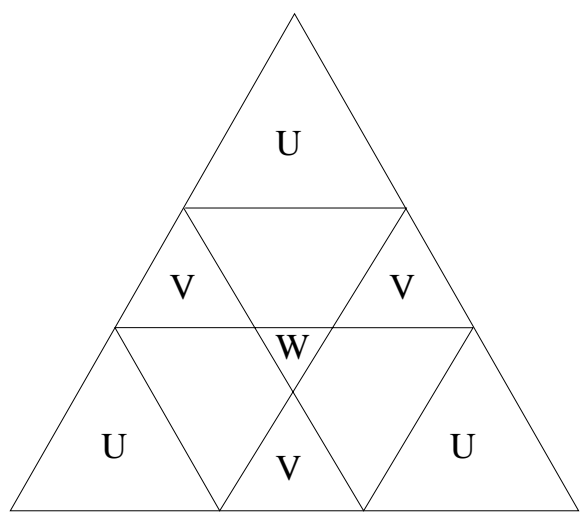

Figure 3: An affine nested fractal

We consider the fractal to be embedded in $\mathbb{R}^{2}$ and hence its boundary is the unit triangle. We note that the boundary dimension in the resistance metric is given by $d_{b}=S_{b}$ where $2 \rho_{1}^{-S_{b}}+$ $\rho_{2}^{-S_{b}}=1$.

Corollary 5.7 In the non-lattice case when $\log \rho_{1} / \log \rho_{2} \notin \mathbb{Q}$ we have a constant $K$ such that

$$
\lim _{t \rightarrow 0} t^{-\left(d_{f}-d_{b}\right) / d_{w}} E_{F}(t)=K .
$$

In the lattice case when $\log \rho_{1} / \log \rho_{2} \in \mathbb{Q}$ we have a periodic function $\varphi(t)$ such that

$$
E_{F}(t)=t^{\left(d_{f}-d_{b}\right) / d_{w}}(\varphi(-\log t)+o(1)), \quad t \rightarrow 0 .
$$

We end with some remarks. 
Remark 5.8 1. The Sierpinski gasket result we obtained directly has a sharper error estimate than that given in this general theorem. Similarly for other specific examples the error estimate may be improved.

2. For higher dimensional affine nested fractals it is straightforward to treat the case where it is only the intersection of the fractal with the face of the tetrahedron or simplex which is maintained at temperature 1 .

\section{References}

[1] M.T. Barlow, Diffusions on fractals, in Lectures in Probability Theory and Statistics: Ecole d'été de probabilités de Saint-Flour XXV (Lect. Notes Math., vol. 1690), Springer, New York, 1998.

[2] M.T. Barlow and R.F. Bass, Construction of Brownian motion on the Sierpinski carpet, Ann Inst H. Poincare, 25 (1989), 225-257.

[3] M.T. Barlow and R.F. Bass, On the resistance of the Sierpinski carpet, Proc. Roy. Soc. London Ser. A 431 (1990), 345-360.

[4] M.T. Barlow and R.F. Bass, Transition density estimates for Brownian motion on the Sierpinski carpet, Probab. Theory Relat. Fields, 91 (1992), 307-330.

[5] M.T. Barlow and R.F. Bass, Brownian motion and harmonic analysis on Sierpinski carpets, Canad. J. Math., 51 (1999), 673-744.

[6] M.T. Barlow, R.F. Bass and T. Kumagai, Stability of parabolic Harnack inequalities on metric measure spaces, J. Math. Soc. Japan, 58 (2006), 485-519.

[7] M.T. Barlow, R.F. Bass, T. Kumagai and A. Teplyaev, Uniqueness for Brownian motion on the Sierpinski carpet. In Preparation

[8] M.T. Barlow and J. Kigami, Localized eigenfunctions of the Laplacian on p.c.f. self-similar sets, J. London Math. Soc. 56 (1997), 320-332.

[9] M.T. Barlow and E.A. Perkins, Brownian motion on the Sierpinski gasket, Probab. Theory Related Fields 79 (1988), 543-623.

[10] M. van den Berg, Heat content and Brownian motion for some regions with a fractal boundary, Probab. Theory Related Fields, 100 (1994), 439-456.

[11] M. van den Berg, Heat equation on the arithmetic von Koch snowflake. Probab. Theory Related Fields, 118 (2000), 17-36.

[12] M. van den Berg and P.B. Gilkey, A comparison estimate for the heat equation with an application to the heat content of the s-adic von Koch snowflake, Bull. London Math. Soc., 30 (1998), 404-412.

[13] M. van den Berg and F. den Hollander, Asymptotics for the heat content of a planar region with a fractal polygonal boundary, Proc. London Math. Soc. 78 (1999), 627-661. 
[14] P.J. Fitzsimmons, B.M. Hambly and T. Kumagai, Transition density estimates for Brownian motion on affine nested fractals, Comm. Math. Phys. 165 (1994), 595-620.

[15] J. Fleckinger, M. Levitin and D. Vassiliev, Heat equation on the triadic von Koch snowflake: asymptotic and numerical analysis, Proc. London Math. Soc. (3) 71 (1995), 372-396.

[16] M. Fukushima and T. Shima, On a spectral analysis for the Sierpiński gasket, Potential Anal. 1 (1992), 1-35.

[17] B.M. Hambly, T. Kumagai, S. Kusuoka and X.Y. Zhou, Transition density estimates for diffusion processes on homogeneous random Sierpinski carpets, J. Math. Soc. Japan 52 (2000), $373-408$.

[18] B.M. Hambly and S.O. Nyberg, Finitely ramified graph directed fractals, spectral asymptotics and the multidimensional renewal theorem, Proc. Edin. Math. Soc., 46 (2003), 1-34.

[19] N. Kajino, Cell counting dimension and spectral asymptotics for Laplacians on self-similar sets, Preprint (2008)

[20] J. Kigami, Analysis on fractals, Cambridge Univ. Press, Cambridge, 2001.

[21] J. Kigami and M.L. Lapidus, Weyl's problem for the spectral distribution of the Laplacian on P.C.F. self-similar fractals, Commun. Math. Phys. 158 (1993) 93-125.

[22] T. Kumagai, Estimates of transition densities for Brownian motion on nested fractals, Probab. Theory Relat. Fields, 96 (1993), 205-224.

[23] T. Kumagai, Short time asymptotic behaviour and large deviation for Brownian motion on some affine nested fractals, Publ. Res. Inst. Math. Sci., 33 (1997), 223-240.

[24] S. Kusuoka and X.Y. Zhou, Dirichlet forms on fractals: Poincaré constant and resistance, Probab. Theory Related Fields 93 (1992), 169-196.

[25] K-S. Lau, J. Wang and C-H. Chu, Vector-valued Choquet-Deny theorem, renewal equation and self-similar measures, Studia Math. 117 (1995), 1-28.

[26] M. Levitin and D. Vassiliev, Spectral asymptotics, renewal theorem and the Berry conjecture for a class of fractals, Proc. London. Math. Soc. 72, (1996), 188-214.

[27] T. Lindstrøm, Brownian motion on nested fractals, Memoirs Amer. Math. Soc. 420, 83, (1990).

[28] R.D. Mauldin and S.C. Williams, Hausdorff dimension in graph directed constructions. Trans. Amer. Math. Soc. 309 (1988), 811-829.

[29] R. Rammal and G. Toulouse. Random walks on fractal structures and percolation clusters, J. Phys. Lett. 44 (1983), L13-122.

[30] C. Sabot, Spectral properties of self-similar lattices and iteration of rational maps, Mem. Soc. Math. Fr. (N.S.) No. 92 (2003). 